\title{
Introduction to the Special Issue for the 11th Triennial Invitational Choice Symposium
}

\author{
Simon J. Blanchard ${ }^{1} \cdot$ Debora V. Thompson $^{1}$ \\ Published online: 29 October 2020 \\ (C) Springer Science+Business Media, LLC, part of Springer Nature 2020
}

From May 29, 2019, to June 2, 2019, in Cambridge, Maryland, Georgetown University hosted the 11th edition of the Triennial Invitational Choice Symposium. The symposium consisted of 25 parallel workshops, each composed of 10-15 participants and each focused on one topic related to human choice behavior and individual decisionmaking. Workshop participants included not only marketing academics but also scholars from computer science, economics, law, medicine, statistical, environmental studies, and transportation. It also included many industry participants and policymakers. All committed to spending three entire days holding in-depth discussions and outlining areas for future research.

Marketing Letters has a long history of supporting the dissemination of novel ideas and stimulating discussion on emerging topics (Golder et al. 2017). Some of Marketing Letters' most impactful articles have come directly from the prior editions of the choice symposium (e.g., Arora et al. 2008; Ben-Akiva et al. 2002; Carson et al. 1994; Johnson et al. 2012). As such, we were thrilled to continue this tradition and invite the co-chairs of the 25 workshops to submit short articles for a special issue at Marketing Letters. After a peer-review process, we accepted ten manuscripts that explore important facets of choice behavior in a variety of domains such as digital voice assistants, transportation decisions, loyalty programs, and machine learning, among others. These articles offer rich new theoretical and practical insights and identify promising new research opportunities. We hope they will spur discussion and stimulate ambitious new projects related to human choice behavior.

Simon J. Blanchard and Debora V. Thompson

11th Triennial Invitational Choice Symposium Co-chairs

Marketing Letters Special Issue Co-editors

Simon J. Blanchard

Simon.Blanchard@georgetown.edu

$\checkmark$ Debora V. Thompson

Debora.Thompson@georgetown.edu

1 McDonough School of Business, Georgetown University, Washington, DC, USA 
Articles in this Special Issue:

- Bhargava et al. (2020), Platform data strategy (https://link.springer.com/article/10. 1007/s11002-020-09539-3)

- Dellaert et al. (2020), Consumer decisions with artificially intelligent voice assistants (https://link.springer.com/article/10.1007/s11002-020-09537-5)

- Feinberg et al. (2020), Choices in networks: a research framework (https://link. springer.com/article/10.1007/s1 1002-020-09541-9)

- Hagen et al. (2020), How can machine learning aid behavioral marketing research? (https://link.springer.com/article/10.1007/s11002-020-09535-7)

- Haruvy et al. (2020), Fundraising design: key issues, unifying framework, and open puzzles (https://link.springer.com/article/10.1007/s11002-020-09534-8)

- Mormann et al. (2020), Time to pay attention to attention: using attention-based process traces to better understand consumer decision-making (https://link.springer. com/article/10.1007/s11002-020-09520-0)

- Proserpio et al. (2020), Soul and machine (learning) (https://link.springer.com/ article/10.1007/s11002-020-09538-4)

- Stourm et al. (2020), Refocusing loyalty programs in the era of big data: a societal lens paradigm (https://link.springer.com/article/10.1007/s11002-020-09523-x)

- Tomaino et al. (2020), Mobility as a service (MaaS): the importance of transportation psychology (https://link.springer.com/article/10.1007/s11002-020-09533-9)

- Wertenbroch et al. (2020), Autonomy in consumer choice (https://link.springer. com/article/10.1007/s11002-020-09521-z)

\section{References}

Arora, N., Dreze, X., Ghose, A., Hess, J. D., Iyengar, R., Jing, B., Joshi, Y., Kumar, V., Lurie, N., Neslin, S., \& Sajeesh, S. (2008). Putting one-to-one marketing to work: personalization, customization, and choice. Marketing Letters, 19(3), 305-321.

Ben-Akiva, M., McFadden, D., Train, K., Walker, J., Bhat, C., Bierlaire, M., Bolduc, D., Boersch-Supan, A., Brownstone, D., Bunch, D. S., \& Daly, A. (2002). Hybrid choice models: progress and challenges. Marketing Letters, 13(3), 163-175.

Carson, R. T., Louviere, J. J., Anderson, D. A., Arabie, P., Bunch, D. S., Hensher, D. A., Johnson, R. M., Kuhfeld, W. F., Steinberg, D., Swait, J., \& Timmermans, H. (1994). Experimental analysis of choice. Marketing Letters, 5(4), 351-367.

Golder, P. N., Jap, S., \& Steckel, J. H. (2017). The future of Marketing Letters. Marketing Letters, 28(3), 335339.

Johnson, E. J., Shu, S. B., Dellaert, B. G., Fox, C., Goldstein, D. G., Häubl, G., Larrick, R. P., Payne, J. W., Peters, E., Schkade, D., \& Wansink, B. (2012). Beyond nudges: tools of a choice architecture. Marketing Letters, 23(2), 487-504.

Publisher's note Springer Nature remains neutral with regard to jurisdictional claims in published maps and institutional affiliations. 